\title{
Evolving Convolutional Neural Networks for Glaucoma Diagnosis
}

\author{
Alan Lima ${ }^{1}$, Lucas B. Maia ${ }^{1}$, Pedro Thiago Cutrim dos Santos ${ }^{1}$, Geraldo Braz Junior ${ }^{1}$, \\ João D. S. de Almeida ${ }^{1}$ and Anselmo C. de Paiva ${ }^{1}$ \\ ${ }^{1}$ Computer Applied Group \\ Federal University of Maranhão (UFMA) \\ São Luís - MA - Brasil \\ \{alanlima, lucas.maia, thiagocutrim, geraldo, jdallyson, paiva\}@nca.ufma.br
}

\begin{abstract}
Glaucoma is an ocular disease that causes damage to the eye's optic nerve and successive narrowing of the visual field in affected patients which can lead the patient, in advanced stage, to blindness. This work presents a study on the use of Convolutional Neural Networks (CNNs) for the automatic diagnosis through eye fundus images. However, building a perfect CNN involves a lot of effort that in many situations is not always able to achieve satisfactory results. The objective of this work is to use a Genetic Algorithm (GA) to optimize CNNs architectures through evolution that can helps in glaucoma diagnosis using eye's fundus image from RIM-ONE-r2 dataset. Our partial results demonstrate satisfactory results after training the best individual chosen by GA with the achievement of an accuracy of $91 \%$.
\end{abstract}

\section{Introduction}

Glaucoma is one of the most common causes of blindness and is expected to affect around 80 million people by 2020 [Chen et al. 2015]. It is a chronic eye disease that can lead to vision loss, in which the optic nerve is progressively damaged due to the increase of intra-ocular pressure (IOP). The symptoms are only detected when the disease is in advanced stage. Although glaucoma cannot be cured, its progression may be slowed down by IOP treatment. Early detection of glaucoma with the assistance of eye fundus images is becoming important because it can let the specialists act against the disease in order to give the patient a better quality of life [Chen et al. 2015].

The technique of eye fundus image acquisition is one of the main and popular ways for glaucoma diagnosis because it is non-invasive. The health care professional uses only one camera capable of acquire important structures from the eye fundus, such as, the optic disc (OD) and blood vessels. These structures are able to provide us useful information for the diagnosis of glaucoma.

In [Chen et al. 2015] is mentioned that among the techniques studied for the diagnosis of glaucoma, Vertical Cup to Disc Ratio (CDR - measure that compares the diameter of the cup region with the total diameter of the OD) is widely used by health care professionals. In addition to this, the disc diameter, ISNT rule (normal eyes show a characteristic configuration for CUP thickness of inferior $\geq$ superior $\geq$ nasal $\geq$ temporal) and peripapillary atrophy (PPA) are also techniques that use OD region, which is the place where the ganglion cell axon exit of the eye to form the optic nerve, through which visual information from the photo-receptors is transmitted to the brain. 
So, different approaches have been developed based on analysis of OD's texture. The work of [de Sousa et al. 2017] presents a methodology based on textures features to suggest a diagnosis of glaucoma from eye fundus images of the optic disc using the Local Binary Pattern (LBP) to represent the optic disc region and geostatistical functions to describe texture patterns. The obtained texture features were classified with Support Vector Machine. As well, the in work of [Araújo et al. 2017] is proposed a method for diagnosis of glaucoma in fundus images using the Shannon and McIntosh diversity indexes as descriptors of texture patterns and support vector machine (SVM) for classification.

In this work, a convolutional neural network $(\mathrm{CNN})$, which has been taking great visibility in the last years, is used to classify images between glaucoma and normal. According to [Sevastopolsky 2017], in addition to being a technique that reduces the efforts of work, to be automated, the great advantage of the use of the CNNs is in the universality, since the same network generally can recognize several patterns in different images and objects through the base features automatically extracted.

However, [Miikkulainen et al. 2017] emphasizes that the success of CNNs depends on finding the best architecture to fit the task, where, in many situations that architecture was constructed after many experiments with complex topologies and hundreds of hyperparameters, an exhaustive hand work with unexpected results. In this scenario emerges the genetic algorithms (GAs), a neuroevolution technique that have been applied successfully for three decades to sequential decision tasks. Examples of good CNN's aplications combined with GAs are [Xie and Yuille 2017, Sun et al. 2017].

Combine with CNNs, GAs are a natural choice mainly due to crossover technique by recombining parts of existing neural networks to find better ones, as well, mutation and selection techniques responsible for choose of the best individual, in our case, the best architecture [Miikkulainen et al. 2017]. Some researchers are studying these techniques in order to take advantage of their discriminative capacity to achieve better results [David and Greental 2017].

The objective of this work is to use a GA to evolve from a simple CNN architecture a robust model to classify eye fundus images in normal or glaucoma, using the $\mathrm{r} 2$ version of the RIM-ONE dataset. The contribution of this work consists in the analysis of performance of the GA to optimize the parameters and architecture of a CNN for characterization of optic disc region and its applicability for glaucoma detection and provide a method that capture precisely fundus features in order to recognize pathologies.

The paper is organized as follows. Section 2 presents the proposed methodology for the development of this work, informing the techniques used in pre-processing, how the GA was developed and CNN was found for the classifying work. Section 3 reports the partial results achieved after the application of the proposed methodology. Section 4 presents conclusions and future works.

\section{Methodology}

The methodology of this work is divided into three stages: pre-processing, run of GA in order to find the best CNN architecture and run of the CNN for glaucoma classification. Figure 1 presents the steps of the proposed method.

The RIM-ONE [Fumero et al. 2011], used in this study, is a public and available 


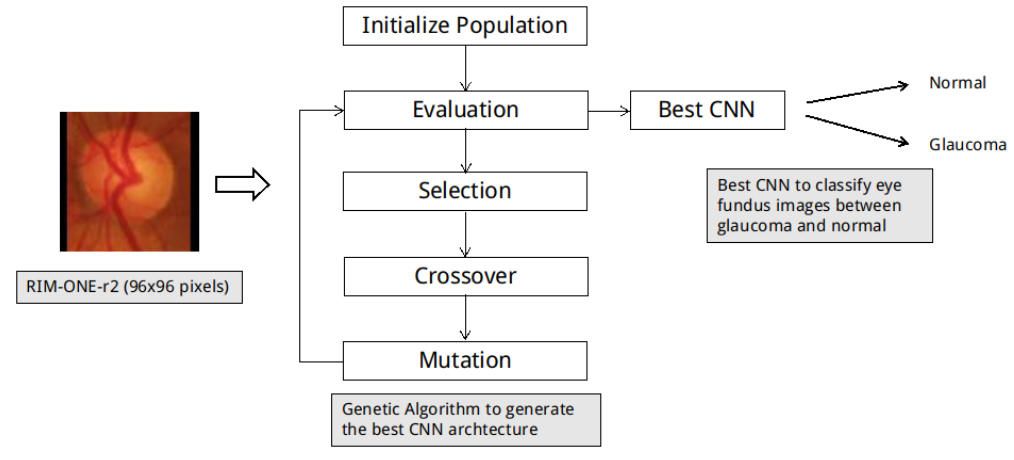

Figure 1. The pipeline of our proposed method

dataset with images in the RGB (Red, Green and Blue) format of the eye fundus image and it was developed to be a reference for the design of segmentation algorithms of the optic nerve.

In our experiments we used the version $\mathrm{r} 2$ of RIM-ONE dataset, which has images of normal class and "glaucoma and suspect" class. The dataset has 455 images, which 255 belongs to the normal class and 200 belongs to the "glaucoma and suspect" class.

\subsection{Pre-processing}

The images from RIM-ONE-r2 were acquired with different widths and heights. The unique pre-processing technique used in this work was resize all images to 96x96 pixels with maintenance of the aspect ratio, by filling with black pixels, a technique known as zero-padding, which consists of zeroing in the edge of the input volume preserving the format of the images, thus being beneficial to an accurate classification [Zheng et al. 2016].

The value of $96 \times 96$ pixels was acquired empirically since another size (28x28, $32 \times 32,64 \times 64$ pixel) were tested with poor results. Greater than $96 \times 96$ pixels was also tested but our GPU (Nvidia GeForce GTX 1060) memory could not support those tests, outputting run out memory message error.

In this version of RIM-ONE dataset the complete image provided by the dataset was used as input ROI (region of interest) for our method. In this approach all information beyond the internal content of the OD was considered, such as, the excavation, macula, and blood vessels.

\subsection{Genetic Algorithm and Training}

This work uses an approach for the development of automatic CNN architectures. It is based on the neuroevolution technique known as NEAT (NeuroEvolution of Augmenting Topologies) [Stanley and Miikkulainen 2002]. The NEAT technique performs the optimization of components, topologies and hyperparameters. The effectiveness of evolved networks is determined on the basis of how well they can be trained, through gradient descent, to perform the task of classification.

The genetic algorithm is a metaheuristic inspired by the process of natural selection. It was proposed by John Holland (1975) and are commonly used to generate high-quality solutions to optimization and search problems by relying on bio-inspired operators such as mutation, crossover and selection [Xie and Yuille 2017]. 
The GA was evaluated by training each CNN generated over a same dataset split in: train data with 227 images, validation data with 92 images, test data with 136 images. Also, this step ran over 200 epochs, using a bath size of 16. To compile each model was used Adam function as optimizer and for loss function was used binary cross-entropy due to our problem be a binary classification problem.

In order to facilitate the development and to accelerate the implementation, all processes were performed in Python with Keras API [Chollet 2015].

To start our GA, an initial population with 20 individuals is created randomly. This population should have at least one layer (with probability of having other layers) with batch normalization, convolution, Relu activation and 50\% of having dropout. Each individual (chromosome) is created in a chain of letters representing one specific layer, as described in Table 1.

Table 1. Example of an chromosome. b: batch normalization; c: convolution; $r$ : relu activation; d: dropout

$$
\begin{array}{|l|l|l|l|}
\hline \mathrm{b} & \mathrm{c} & \mathrm{r} & \mathrm{d} \\
\hline
\end{array}
$$

The evaluation function determines the fitness of an individual [Xie and Yuille 2017]. Because of our propose concerns on solving image classification tasks, the classification validation loss is the best strategy to assign their fitness (fitness $=1 /$ val_loss $)$. In this case, on each generation the best individual will be chosen with the minor validation loss after the training of this individual run.

Selection will return a random new parent using tournament selection criteria, selecting the top performer to carry over to the next generation, and to mate with the rest of the population. Selection happens for each child in the new population [Yadav and Soha 2017].

The crossover is the technique that will selected the parents of an individual to mix and match their chromosomes to produce two new children. In our case, parents should have equal length of layers because the crossover happen in single point model and this point is chosen randomly, respecting the structural of CNN. The probability of crossover happen in our GA is set to 30\%. [Xie and Yuille 2017].

Mutation, according [Xie and Yuille 2017], allows the algorithm to introduce diversity into the population, expanding the opportunity to search unexplored areas in the search space for fitter solutions. Mutation is implemented by giving each element in the chromosome array a probability of following scenarios be randomly drawn: removing or adding convolution blocks; adding or removing dropout; changing the order of convolution blocks; changing the inputs of convolution blocks; changing the outputs of the big block; changing the order of big blocks.

To evaluate each CNN, the training process was used on train set data, the validate process on validation set data and test process on test set data of RIM-ONE-r2.

\section{Partial Results}

The accuracy was used as criteria to assess the proposed model to classify the glaucoma, since the database is almost balanced. After GA ran 20 generations (stop condition) over 22 hours, it could select the best CNN architecture (25 layers) as described in Figure 2. 


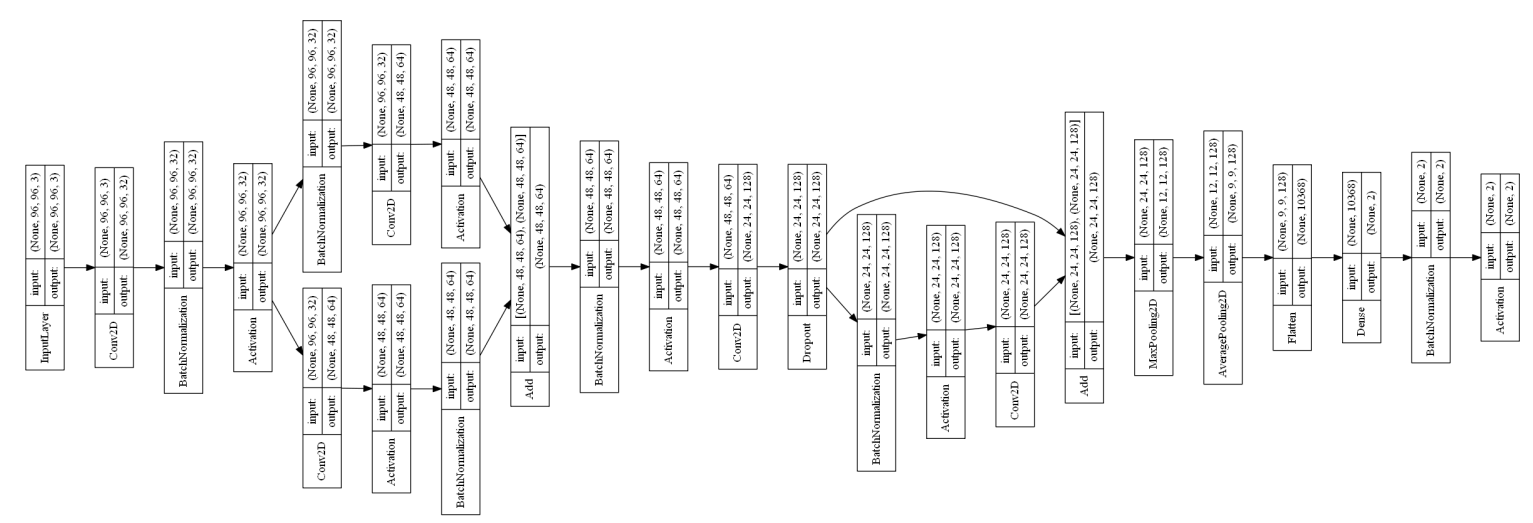

Figure 2. Best individual found by GA: CNN with 25 layers

This architecture reaches an accuracy of $91 \%$ on test dataset (136 images - 76 normal class and 60 "glaucoma and suspect" class), as showed in Tab. 2. It means that the model could discriminate between the glaucoma and normal in a big portion of test set data, also, the loss error is small after running the test set data through the trained network.

Table 2. GA evolution over $20^{\mathrm{a}}$ generations

\begin{tabular}{|l|c|c|c|c|c|c|c|c|c|c|}
\hline Gen & $1^{\mathrm{a}}$ & $2^{\mathrm{a}}$ & $3^{\mathrm{a}}$ & $4^{\mathrm{a}}$ & $5^{\mathrm{a}}$ & $\mathbf{6}^{\mathbf{a}}$ & $7^{\mathrm{a}}$ & $8^{\mathrm{a}}$ & $\mathbf{9}^{\mathbf{a}}$ & $10^{\mathrm{a}}$ \\
\hline LOSS & 0.27 & 0.25 & 0.26 & 0.26 & 0.27 & $\mathbf{0 . 2 5}$ & 0.26 & 0.29 & $\mathbf{0 . 2 5}$ & 0.26 \\
\hline ACC $(\%)$ & 0.85 & 0.88 & 0.90 & 0.89 & 0.89 & $\mathbf{0 . 9 1}$ & 0.88 & 0.90 & $\mathbf{0 . 9 1}$ & 0.87 \\
\hline \hline Gen & $11^{\mathrm{a}}$ & $12^{\mathrm{a}}$ & $13^{\mathrm{a}}$ & $\mathbf{1 4}^{\mathrm{a}}$ & $15^{\mathrm{a}}$ & $16^{\mathrm{a}}$ & $17^{\mathrm{a}}$ & $18^{\mathrm{a}}$ & $\mathbf{1 9}^{\mathrm{a}}$ & $20^{\mathrm{a}}$ \\
\hline LOSS & 0.28 & 0.25 & 0.25 & $\mathbf{0 . 2 2}$ & 0.23 & 0.26 & 0.27 & 0.25 & $\mathbf{0 . 2 6}$ & 0.27 \\
\hline ACC $(\%)$ & 0.85 & 0.88 & 0.90 & $\mathbf{0 . 8 9}$ & 0.89 & 0.89 & 0.89 & 0.90 & $\mathbf{0 . 9 2}$ & 0.88 \\
\hline
\end{tabular}

The best preliminary result was found in 6th generation. There was a great improvement of results between the 1st generation, with $85 \%$ and 9 th with $91 \%$. Showing the efficacy of the method when estimating the depth of CNN architecture and the hyperparametres.

After the 6th generation, the GA tried to improve those results and reached the same value in 9th generation. It's important to mention that in generations 14th and 19th the GA could reach the best loss error and the best accuracy score, respectively, but, for ours goals, the best individual need be a CNN with a low loss error and high accuracy score at same time.

\section{Conclusion and Future Works}

The main objective of this under construction study was a classification approach of normal and glaucomatous eye fundus images was presented using a $\mathrm{CNN}$ architecture evolved by GA. The evolved CNN was trained on RIM-ONE-r2 dataset. The last layers of the CNN were used as traditional classified. The obtained results proved the effectiveness of the developed approach, where the generated architecture, together with the parameters selected from neuroevolutionary techniques obtained from the dataset of images was capable to constructed a CNN with 25 layers and reach an accuracy of $91 \%$.

In future work, new training should be performed in the GA to achieve a better optimization of the parameters and to increase the accuracy in the training. We intend to 
use a larger set of images and other available datasets to verify the effectiveness of the use of GA and CNN in the classification of glaucoma. Also, try another batch size and optimize functions in training process.

\section{References}

Araújo, J. D. L., de Paiva, A. C., de Almeida, J. D. S., Neto, O. P. S., de Sousa, J. A., Silva, A. C., and Júnior, G. B. (2017). Diagnóstico de glaucoma em imagens de fundo de olho utilizando os Índices de diversidade de shannon e mcintosh. XXXVII Congresso da Sociedade Brasileira de Computação - $17^{\circ}$ WIM - Workshop de Informática Médica, pages 1873-1882.

Chen, X., Xu, Y., Wong, D. W. K., Wong, T. Y., and Liu, J. (2015). Glaucoma detection based on deep convolutional neural network. In 2015 37th Annual International Conference of the IEEE Engineering in Medicine and Biology Society (EMBC), pages $715-718$.

Chollet, F. (2015). Keras. https://github.com/fchollet/keras. [Online; accessed 15-March-2017].

David, E. and Greental, I. (2017). Genetic algorithms for evolving deep neural networks. CoRR, abs/1711.07655.

de Sousa, J. A., de Paiva, A. C., Sousa de Almeida, J. D., Silva, A. C., Junior, G. B., and Gattass, M. (2017). Texture based on geostatistic for glaucoma diagnosis from fundus eye image. Multimedia Tools and Applications, 76(18):19173-19190.

Fumero, F., Alayon, S., Sanchez, J. L., Sigut, J., and Gonzalez-Hernandez, M. (2011). Rim-one: An open retinal image database for optic nerve evaluation. In 2011 24th International Symposium on Computer-Based Medical Systems (CBMS), pages 1-6.

Miikkulainen, R., Liang, J. Z., Meyerson, E., Rawal, A., Fink, D., Francon, O., Raju, B., Shahrzad, H., Navruzyan, A., Duffy, N., and Hodjat, B. (2017). Evolving deep neural networks. CoRR, abs/1703.00548.

Sevastopolsky, A. (2017). Optic disc and cup segmentation methods for glaucoma detection with modification of u-net convolutional neural network. Pattern Recognition and Image Analysis, 27(3):618-624.

Stanley, K. O. and Miikkulainen, R. (2002). Evolving neural networks through augmenting topologies. Evolutionary Computation, 10(2):99-127.

Sun, Y., Xue, B., and Zhang, M. (2017). Evolving deep convolutional neural networks for image classification. CoRR, abs/1710.10741.

Xie, L. and Yuille, A. L. (2017). Genetic CNN. CoRR, abs/1703.01513.

Yadav, S. L. and Soha, A. (2017). Comparative study of different selection techniques in genetic algorithm. International Journal of Engineering, Science and Mathematics, $6(3)$.

Zheng, L., Zhao, Y., Wang, S., Wang, J., and Tian, Q. (2016). Good practice in CNN feature transfer. CoRR, abs/1604.00133. 